\title{
Preparation of Polyimide Gas Separation Membrane Made by Ion Implantation Treatment
}

\author{
Teppei Tezuka, ${ }^{1}$ Tomohiro Kobayashi, ${ }^{2}$ Kenji Fukui, ${ }^{1}$ Shoji Nagaoka, ${ }^{1}$ Yoshiaki Suzuki, ${ }^{2}$ \\ Masaya Iwaki, ${ }^{2}$ and Hiroyoshi Kawakami ${ }^{1 *}$ \\ 1: Department of Applied Chemistry, Tokyo Metropolitan University, Hachioji, Tokyo 192-0397, Japan \\ 2: The Institute of Physical and Chemical Research, 2-1 Hirosawa, Wako, Saitama 351-0198, Japan
}

Keywords: polyimide, ion implantation, gas separation

\section{Introduction}

The gas separation process using polymer membranes has received much attention, because the membrane systems provide better energy efficiency than conventional separation methods $[1,2]$. Many polymers have been synthesized to control the gas permeability and selectivity of the polymer membranes, because the ability to achieve such control provides a good understanding of the relationships between the chemical structure of polymers and the gas permeation property [3.4]. However, many studies of the structure/permeability relationships of polymer membranes have led to the trade-off correlations between the gas permeability and selectivity, which have become a major problem in realizing a gas separation process using polymer materials. Therefore, an important objective for the gas separation membrane is the development of new polymer membranes combining high gas permeability and selectivity.

Recently, we reported that an asymmetric polyimide membrane with an ultrathin and defect-free carbonized skin layer prepared by the modification of the skin surface using ion-beam irradiation had a high gas permeability and selectivity [5-7]. It is well known that ion-beam irradiation can directly modify the surface of a membrane and that the polymer surface irradiated under a high ion fluence is carbonized $[8,9]$. The asymmetric membrane with the carbonized skin layer was characterized by its toughness and good mechanical properties, and we demonstrated that the ultrathin and defect-free carbonized skin layer was able to realize both a high gas permeability and selectivity.
On the other hand, plasma-based ion implantation (PBII) is a recent technique developed for modification of material surface and has some advantages as compared to ion-beam irradiation [10]. In particular, PBII can offer the treatment of large areas and three-dimensional materials with complex shapes. There are some reports for gas-barrier materials prepared using PBII $[11,12]$. However, there have been few papers reporting the gas separation polymer membranes prepared using PBII.

In this paper, we describe the gas permeability of the polyimide membranes prepared by PBII. The membrane, which used to synthesize a novel gas separation membrane combining high gas permeability and selectivity, exhibits an asymmetric structure consisting of a carbonized skin layer and a polymeric porous substructure. We prepared an asymmetric polyimide membrane with a thin skin layer carbonized by PBII. The asymmetric membrane with the carbonized skin layer is characterized by being tough and having a good mechanical property, and we consider that the ultrathin and defect-free carbonized skin layer would realize both a high gas permeability and selectivity.

The objective of our study is to fabricate a novel asymmetric polyimide membrane with an ultrathin and defect-free carbonized skin layer. In this preliminary study, the gas permeances of the asymmetric polyimide membranes implanted with $2.5 \mathrm{kV} \mathrm{He}{ }^{+}$have been measured using a high vacuum apparatus with a Baratron absolute pressure gauge at $76 \mathrm{cmHg}$. Additionally, the temperature dependence of the gas permeability through the asymmetric membrane is also 
discussed in this study. The present paper will first address the gas permeability of the asymmetric polyimide membranes prepared by PBII

\section{Experimental Section}

\subsection{Materials}

2,2'-Bis(3,4-dicarboxyphenyl)hexafluoro propane dianhydride (6FDA) was purchased from the Central Glass Co., (Saitama, Japan) and purified by sublimation prior to use. The diamine monomer was 2,2'-bis(4-aminophenyl)hexafluoropropane (6FAP), which was purchased from the Central Glass Co., (Saitama, Japan) and recrystallized twice in ethanol prior to use.

The polyimide, 6FDA-6FAP, was synthesized by chemical imidization of the poly(amic acid) precursors as reported in the literature $[13,14]$. The structures of 6FDA-6FAP are shown in Figure 1. The synthesized 6FDA-6FAP had an $\mathrm{Mw}$ of $4.8 \times 10^{5}$ with a polydispersity index of 1.7.

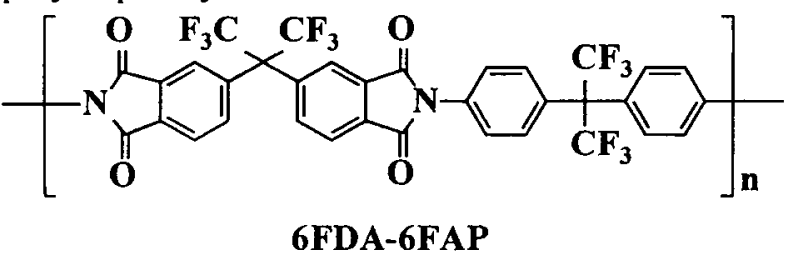

Figure 1. Chemical structure of polyimide.

\subsection{Preparation of Asymmetric Polyimide Membranes}

The asymmetric polyimide membrane, membranes with a skin layer, approximately 116 $\mathrm{nm}$, were prepared by a dry/wet phase inversion process $[15,16]$.

\subsection{Plasma-based Ion Implantation (PBII) for Asymmetric Membranes}

The asymmetric polyimide membranes (25 $\mathrm{mm}$ in diameter) were irradiated with $\mathrm{He}$ ions using PBII for 15 to $150 \mathrm{sec}$ at $10 \mathrm{mTorr}$. PBII was performed by applying pulsed-RF $(13.56 \mathrm{MHz}$, $500 \mathrm{~W}, 30 \mathrm{msec})$ and pulsed-DC $(-2.5 \mathrm{kV}, 10 \mu \mathrm{sec})$ fields alternately to the electrically floated sample holder at a cycle of $2 \mathrm{kHz}$.

\subsection{Surface Characterization of Asymmetric Membranes}

The cross sections of the asymmetric polyimide membranes were observed using a scanning electron microscope (SEM, JXP-6100P, JEOL, Tokyo, Japan).

The surface change in the polyimide membranes before and after PBII was measured by laser Raman spectroscopy (LabRam, Jobin Yvon, Tokyo, Japan). A square Ge crystal in an ATP objective was used, and sixty-four scans of $2 \mathrm{~cm}^{-1}$ resolution were averaged to achieve a sufficient signal-to-noise ratio. The Raman spectra were observed using an He-Ne laser of $632.817 \mathrm{~nm}$.

\subsection{Gas Permeation Measurements.}

Gas permeances of oxygen and nitrogen were measured with a high vacuum apparatus (Rika Seiki, Inc., K-315-H, Tokyo, Japan). The gas permeation measurements of the membranes were carried out at $35^{\circ} \mathrm{C}$ and $76 \mathrm{cmHg}$. The apparent skin layer thickness of the asymmetric polyimide membranes was calculated from

$$
L=\frac{P}{Q}
$$

where $\mathrm{L}[\mathrm{cm}]$ is the apparent skin layer thickness, $\mathrm{P}$ $\left[\mathrm{cm}^{3}\right.$ (STP) $\left.\mathrm{cm} /\left(\mathrm{cm}^{2} \mathrm{sec} \mathrm{cmHg}\right)\right]$ is the gas permeability coefficient measured from the dense polyimide flat membrane, and Q $\left[\mathrm{cm}^{3}(\mathrm{STP}) /\left(\mathrm{cm}^{2}\right.\right.$ $\mathrm{sec} \mathrm{cmHg}$ )] is the gas permeance of the asymmetric polyimide membranes. $\mathrm{L}$ was determined from the oxygen permeability coefficient.

\section{Results and Discussion}

\subsection{Characteristics of Asymmetric Membranes}

(a)

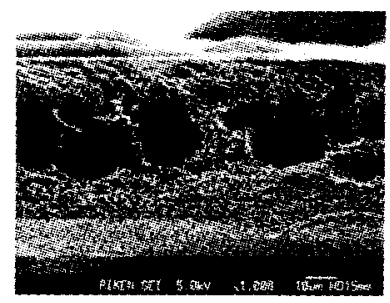

(b)

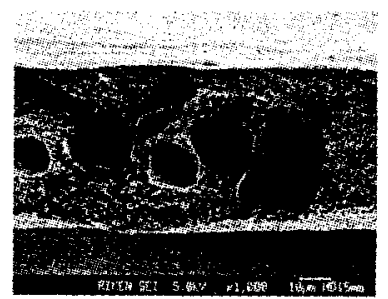

Figure 2. SEM pictures of cross section of asymmetric polyimide membranes: (a) control, (b) $\mathrm{He}^{+}$implantation at $60 \mathrm{sec}$.

Figure 2 shows the results of the SEM observations of the asymmetric polyimide membranes before and after PBII. The cross sectional structure of the asymmetric membrane consisted of an ultrathin skin layer and a porous 


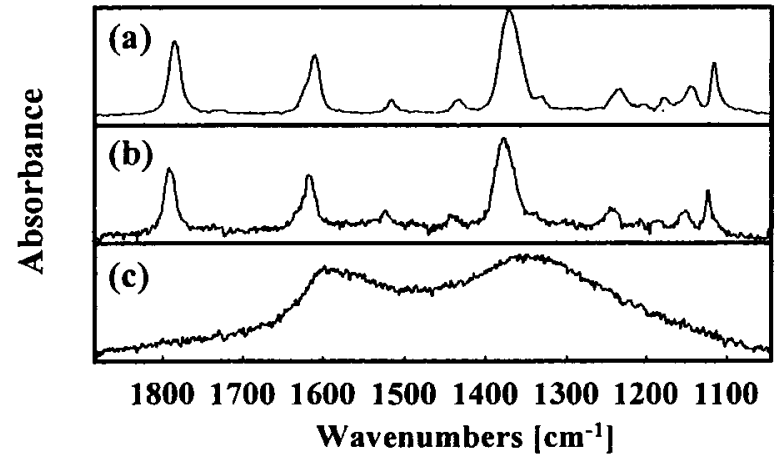

Figure 3. Raman spectra of asymmetric polyimide membranes:

(a) control, (b) $\mathrm{He}^{+}$implantation at $60 \mathrm{sec}$,

(c) $\mathrm{He}^{+}$implantation at $150 \mathrm{sec}$.

substructure characterized by the presence of finger-voids. There was no significant difference in the cross sectional structure of the asymmetric membranes before and after PBII.

Figure 3 shows the Raman spectra of the asymmetric polyimide membranes implanted by a $2.5 \mathrm{kV} \mathrm{He}^{+}$. The treatment time of $\mathrm{He}$ ions were 60 and $150 \mathrm{sec}$. The peaks at 1240,1380, 1620, and $1780 \mathrm{~cm}^{-1}$ correspond to $\mathrm{C}-\mathrm{F}_{3}, \mathrm{C}-\mathrm{N}$, and $\mathrm{C}=\mathrm{C}$ in the aromatic ring, and $\mathrm{C}=\mathrm{O}$, respectively. After the implantation of a $2.5 \mathrm{kV} \mathrm{He}{ }^{+}$for $150 \mathrm{sec}$, these peaks on the polyimide surface disappeared. However, two broad peaks on the polyimide surface irradiated after a $150 \mathrm{sec}$ PBII treatment appeared at around 1360 and $1580 \mathrm{~cm}^{-1}$. These peaks correspond, respectively, to the well-known $\mathrm{D}$ and $\mathrm{G}$ broad bands of disordered graphitic materials, ${ }^{23}$ indicating that the surface on the membrane changed to a carbon-enriched material.

\subsection{Gas Permeation Properties of Asymmetric Membranes}

The depth profiles of the energy loss for $\mathrm{He}^{+}$irradiation at $2.5 \mathrm{kV}$ in the asymmetric polyimide membrane are estimated using a well-established Monte Carlo simulation method (TRIM code). The $\mathrm{He}^{+}$in the polyimide membrane implanted at $2.5 \mathrm{kV}$ completely losses the electronic and nuclear energies at a $116 \mathrm{~nm}$ depth.

The results of the gas permeance and selectivity of the asymmetric polyimide membranes for $\mathrm{O}_{2}$ and $\mathrm{N}_{2}$ at $35^{\circ} \mathrm{C}$ and $76 \mathrm{cmHg}$ are shown in Table 1. The dense polyimide membrane as the control membrane indicated an oxygen permeability coefficient $\left(\mathrm{PO}_{2}\right)$ of $1.48 \times 10^{-9}\left[\mathrm{~cm}^{3}(\mathrm{STP}) \mathrm{cm} /\left(\mathrm{cm}^{2}\right.\right.$
Table 1. Effect of $\mathrm{He}^{+}$implantation on gas transport properties of asymmetric polyimide membranes at $76 \mathrm{cmHg}$ and $35^{\circ} \mathrm{C}$.

\begin{tabular}{cccc}
\hline $\begin{array}{l}\text { Skin layer } \\
\text { thickness (nm) }\end{array}$ & $\begin{array}{l}\text { Treatment } \\
\text { time (sec) }\end{array}$ & $\begin{array}{l}\mathrm{QO}_{2} \\
\text { (GPU) }\end{array}$ & $\mathrm{QO}_{2} / \mathrm{QN}_{2}$ \\
\hline 117 & 0 & 110 & 5.3 \\
109 & 30 & 24 & 7.3 \\
111 & 60 & 13 & 8.2 \\
117 & 150 & 1.2 & 3.1 \\
\hline
\end{tabular}

GPU : $10^{-6}\left[\mathrm{~cm}^{3}(\mathrm{STP}) / \mathrm{cm}^{2} \mathrm{sec} \mathrm{cmHg}\right]$

sec $\mathrm{cmHg})]$ and a $\left(\mathrm{PO}_{2} / \mathrm{PN}_{2}\right)$ of 4.7. The apparent skin layer thickness of the asymmetric polyimide membrane before PBII was calculated from the $\mathrm{PO}_{2}$ of the dense membrane. The mean skin layer thickness calculated from the $\mathrm{PO}_{2}$ was $113 \pm 4 \mathrm{~nm}$. Therefore, $\mathrm{He}$ ions are considered to have penetrated through the skin layer in the asymmetric polyimide membrane.

As shown in Table 1, both the gas permeance and selectivity of the asymmetric polyimide membranes strongly depended on the $\mathrm{He}^{+}$treatment time. The $\mathrm{O}_{2} / \mathrm{N}_{2}$ selectivities of the asymmetric membranes implanted at the $\mathrm{He}^{+}$ treatment time of less than $60 \mathrm{sec}$ increased when compared with the dense $\left(\mathrm{O}_{2} / \mathrm{N}_{2}=4.7\right)$ and asymmetric $\left(\mathrm{O}_{2} / \mathrm{N}_{2}=5.3\right)$ membranes before PBII, which indicates that the permeation of the asymmetric membrane is predominantly carried out by a solution-diffusion mechanism and that the surface skin layer is essentially defect-free. It should be noted that the $\left(\mathrm{O}_{2} / \mathrm{N}_{2}\right)$ selectivity in the asymmetric membrane implanted at $60 \mathrm{sec}$ resulted in 1.5 times increases when compared with that of the asymmetric membrane before PBII. One possibility for the gas selectivity of the asymmetric membrane treated by PBII may be that the gas permeation space, which can provide a high degree of size and shape discrimination between the gas molecules, was formed on the ion-implanted skin surface and suppressed the permeability of $\mathrm{N}_{2}$ rather than that of $\mathrm{O}_{2}$. In contrast, the selectivity of the asymmetric membranes at a $150 \mathrm{sec}$ treatment time decreased. On the other hand, the $\mathrm{O}_{2}$ and $\mathrm{N}_{2}$ permeances in the asymmetric membranes after PBII decreased with an increase in the $\mathrm{He}^{+}$treatment time. 
Table 2. Effect of temperature on $\mathrm{O}_{2} / \mathrm{N}_{2}$ selectivity of asymmetric polyimide membranes at $76 \mathrm{cmHg}$.

\begin{tabular}{ccccc}
\hline $\begin{array}{c}\text { Treatment } \\
\text { time (sec) }\end{array}$ & $15^{\circ} \mathrm{C}$ & $25^{\circ} \mathrm{C}$ & $35^{\circ} \mathrm{C}$ & $45^{\circ} \mathrm{C}$ \\
\hline 0 & 6.2 & 5.7 & 5.3 & 4.9 \\
30 & 8.2 & 7.7 & 7.3 & 6.9 \\
60 & 9.3 & 8.6 & 8.2 & 7.7 \\
150 & 2.7 & 2.9 & 3.0 & 3.1 \\
\hline
\end{tabular}

Table 2 shows the temperature effect on the $\mathrm{O}_{2} / \mathrm{N}_{2}$ selectivities of the asymmetric polyimide membranes before and after PBII. The $\mathrm{O}_{2} / \mathrm{N}_{2}$ selectivities of the asymmetric membrane treated by PBII at $60 \mathrm{sec}$ indicated the largest values. In contrast, the selectivities of the asymmetric membrane at $150 \mathrm{sec}$ were smaller than those of the membrane before PBIl, indicating that the skin layer of the asymmetric membrane might have the pores.

\section{Conclusions}

The above studies have led to some interesting conclusions for the gas permeance and selectivity of the asymmetric polyimide membranes prepared by PBIl. The gas permeances for the membrane showed a decrease with the increasing treatment time. On the other hand, the gas selectivity increased with an increase in the treatment time by $60 \mathrm{sec}$.

It is desirable to prepare a polymer membrane with both a high gas permeability and selectivity for gas separation. The asymmetric polyimide membrane prepared by PBII showed interesting gas permeation results, and we consider that the PBIl would be one of the important membrane fabrication techniques for realizing a high gas permeability and selectivity.

\section{Acknowledgment}

This work was partially supported by grant 16310062 from the Ministry of Education, Culture, Sports, Science and Technology.

\section{References}

[1] A. Thran, G. Kroll, F. Faupel J. Polym. Sci., Poly, Phys. Ed. 37 (1999) 3344.

[2] S. A. Stern, J. Membr. Sci. 94 (1994) 1.

[3] R. L. Burns, W. J. Koros Macromoleclues 36 (2003) 2374.

[4] Y. Dai, M. D. Guiver, G. P. Robertson, Y. S. Kang, K. J. Lee Macromoleclues 36 (2003) 6807.

[5] M. Iwase, A. Sannnomiya, S. Nagaoka, Y. Suzuki, M. Iwaki, H. Kawakami Macromolecules $237(2004) 6892$.

[6] A. Sannomiya, S. Nagaoka, Y. Suzuki, M. Iwaki, H. Kawakami Polymer 47 (2006) 6585.

[7] A. Sannomiiya, K. Fukui, S. Nagaoka, Y. Suzuki, M. Iwaki, H. Kawakami J. Polym. Sci., Part B 45 (2007) 262.

[8] V. Svoricik, E. Arenholz, V. Rybka, V. Hnatowicz Nucl. Instrum. Methods Phys. Res. Sect.B 122 (1997) 663.

[9] A. M. Ektessabi, S. Hakamata Thin Solid Films 621 (2000) 377.

[10] M. Wofhard, M. Subroto Current Science 83-3 (2002) 237..

[11] N. Sakudo, H. Endo, R. Yoneda, Y. Ohmura, N. Ikenaga Surface \& Coatings Technology $196(2005) 394 .$.

[12] S. Okuji, M. Sekiya, M. Nakabayashi, H. Endo, N. Sakudo, K. Nagai Nucl. Instrum. Methods Phys. Res. Sect.B 242 (2006) 353.

[13] H. Kawakami, J. Anzai J. Appl. Poly. Sci. 57 (1995) 789.

[14] H. Kawakami, M. Mikawa J. Membr. Sci. 118 (1996) 223.

[15] H. Kawakami, M. Mikawa J. Appl. Polym. Sci. 62 (1996) 965.

[16] H. Kawakami, M. Mikawa, S. Nagaoka Macromolecules 31 (1998) 6636. 